\title{
The relativistic effects of charged particle with complex structure in zeropoint field
}

\begin{abstract}
A charged particle immersed in the random fluctuating zeropoint field is considered as an oscillator with oscillations at random directions and the stochastic average of all such oscillations may be considered as local complex rotation in complex vector space. The average internal oscillations or rotations of the charged particle reveal the particle extended structure with separated centre of charge and centre of mass. The aim of this short review article is to give an account of the extended particle structure in complex vector space and to study the origin of relativistic effects due to a charged particle motion in the presence of zeropoint field.
\end{abstract}

Volume I Issue 4 - 2018

Kundeti Muralidhar

Department of Physics, National Defence Academy, India

Correspondence: Kundeti Muralidhar, Department of Physics, National Defence Academy, Khadakwasla, Pune-4I I023, Maharashtra, India, Email kundetimuralidhar@gmail.com

Received: April 28, 2018| Published: July 10, 2018

Keywords: special relativity, zeropoint energy, particle mass, spin, geometric algebra

\section{Introduction}

In most of the theoretical studies, a charged particle like electron is normally treated as a point particle and in quantum mechanics the particles behave like waves. The wave particle duality is one of the main features of quantum systems and it led to the development of quantum theory. Merging of quantum mechanics with relativity was initially established by Dirac and in his theory the velocity of electron was found to be equal to the velocity of light. The ambiguity has been clarified with the invention of zitterbewegung motion or internal oscillations of the electron and the cause of such oscillations is attributed to the presence of random fluctuating zeropoint field present throughout universe. Since the internal oscillations occupy certain region of space, the electron must have certain substructure or extended structure. The observed relativistic effects of the particles are derived from the requirement of constancy of light velocity in all inertial frames of reference and the principle of relative motion. The mathematical theory by Einstein elucidates the fact that the structure of space time is compatible with Maxwell's electromagnetic field equations and we treat the particles as point particles. In the following sections we shall study how the consideration of extended structure of charged particles gives the relativistic effects.

The proposal of extended structure of electron stems from the Schrödinger's study of Dirac electron internal oscillations of the order of reduced Compton wavelength. ${ }^{1,2}$ The oscillating motion of electron is known as zitterbewegung and in which an additional position coordinate oscillates with high frequency. This theoretical formulation elucidates the particle internal structure is described by oscillating centre of charge around centre of mass position. In the zitterbewegung study of electron by Kerson Huang ${ }^{3}$ the charge centre of electron moves in a circular fashion about the direction of electron spin with radius of the order of Compton wave length. Similarly, in the classical spinning electron model of Mathisson, ${ }^{4}$ the electron motion is separated into free motion of centre of mass and the internal rotational motion of centre of charge due to zitterbewegung. Mathisson's theory was further extended by Wyssenhoff and Raabe and they suggested that the proper time is connected to the centre of mass motion when observed from an arbitrary frame of reference. ${ }^{5-6}$ Barut and Zanghi ${ }^{7}$ studied the classical analogue of zitterbewegung in the formalism of geometric algebra. They found that the invariant proper time is associated with the centre of mass. In the Hestenes model of Dirac electron ${ }^{8,9}$ the electron spin was considered as the zeropoint angular momentum. The above considerations univocally suggests that an elementary particle (like electron or quark) contains a sub-structure described by point charge rotating in circular motion around centre of mass and the angular frequency of such rotation is equal to the zitterbewegung frequency. Further, the spin angular momentum and the magnetic moment of a charged particle are found to be the manifestation of internal circular motion. The charge centre oscillations are also responsible for deviations in the average path of charged particle. In a different approach, considering the kinematical theory of spinning particles, an extended particle structure was studied by Rivas. ${ }^{10}$

In general, an electron is visualized as a point particle in both quantum mechanics and quantum field theories and efforts to find the size of electron led to a very small size, $\sim 10^{-20} \mathrm{~m}$ in high energy scattering experiments. ${ }^{11}$ The point particle limit of electron, in most of the theoretical approaches is fine and excellent except for the singularity leading to infinite energy and any cut-off procedure leads to a finite structure of electron. However, the high energy scattering experiments neither confirm point particle nature of electron nor its minuscular size. Alternatively, the concept of an extended structure of electron originates from the zitterbewegung motion and such random oscillations are invariably attributed to the presence of zeropoint field throughout universe. The experimental evidence for the extended structure of electron has been found in a recent electron channelling experiment by Gouanère et al. ${ }^{12}$ In this experiment, the electrons in a beam aligned close to the crystal axis are trapped spiralling around a single atomic row and there by the transmission through the crystal is greatly enhanced with reduced scattering. The transmission resonance occurred at energy $80.874 \mathrm{MeV}$. A theoretical explanation for the observed energy was given by Hestenes, ${ }^{9}$ considering helical motion of electron. Further, the signature of the extended electron structure can be seen from the high resolution scanning tunneling microscopy images studied recently by Hofer. ${ }^{13}$

In classical electrodynamics, we consider the universe contains only radiation field in the remote past and it is regarded that at a 
finite time the radiation comes from somewhere and the incoming radiation is ignored or set to zero. However, the universe is filled with ubiquitous zeropoint field and hence the incoming radiation cannot be ignored. Stochastic electrodynamics is a subject which deals with both incoming zeropoint radiation field and classical electrodynamics. ${ }^{14}$ In stochastic electrodynamics, the randomness arises in a similar way as in the statistical mechanics involving the averaging over many microscopic and deterministic degrees of freedom. Stochastic electrodynamics was mainly developed to achieve a reasonable classical approach to the foundations of quantum mechanics and to certain extent quantum electrodynamics. Assuming zeropoint field, a priori several number of quantum phenomena have been studied in the stochastic electrodynamics. A complete account of stochastic electrodynamics was discussed in several reviews. ${ }^{14-17}$ Though the stochastic electrodynamics has been used to explain many interesting results of quantum mechanics, the theory has certain serious drawbacks. In the presence of fluctuating zeropoint filed, a charged particle like electron is considered as an oscillator. The energy of such an oscillating charged massive particle would be divergent if an upper cut-off frequency to the spectrum of zeropoint field is not imposed. The theory is limited only to linear forces but it cannot be applicable to nonlinear forces. However, when a particle oscillates in zeropioint field, it may be assumed that the centre of mass cannot follow the motion of centre of charge similar to a phenomenon that happens in extended charged bodies. Based on this assumption, Rueda and Cavalleri proposed a classical model for the particle in accordance with the zitterbewegung motion of a charged particle containing internal structure. ${ }^{18}$ The vibrations of charge with respect to centre are assumed due to the zeropoint field and a cut-off to the zeropoint field distribution corresponds to the particle extended structure. ${ }^{19,20}$ The oscillations of the charge around the centre of mass point may be considered as rotations on complex plane. The angular momentum of the internal circular motion represents the spin of the particle, the frequency of spin rotation gives the natural cut-off frequency for the zeropoint spectrum which is the maximum frequency radiated (absorbed) by the charged particle in the zitterbewegung interpretation and hence the main drawback of normal stochastic electrodynamics, the problem of divergence is eliminated. It has been shown recently that the introduction of spin into stochastic electrodynamics eliminates all the drawbacks of classical stochastic electrodynamics. $^{21,22}$ In stochastic electrodynamics with spin, Cavalleri et al., ${ }^{21}$ explained several interesting phenomena: stability of elliptical orbits in an atom, narrow spectral lines in stochastic process, interpretation of Aharonov-Bohm effect, explanation for diffraction of electrons etc. In this theory, a charged particle is considered to possess an extended internal structure which is described by circular motion of charge at the speed of light around centre of mass and when the particle moves with certain velocity, the charge centre executes a helical motion. More interestingly, the special relativity is not present at the particle level and it originates naturally from the helical motion of particle charge.

Recently, the role of spin and the internal charged particle structure in complex vector formalism have been studied by the author. ${ }^{23-25}$ In the presence of zeropoint field, an elementary charged particle oscillates in accordance with the oscillations of the random zeropoint field and an average of all such oscillations may be considered as complex rotations and the imaginary part of such complex rotation gives the classical origin of particle spin. ${ }^{26,27}$ It has been shown that the mass of the particle may be interpreted to the zeropoint field energy associated with the local complex rotation or oscillation confined in a region of space of the order of Compton wavelength. When the particle centre of mass is in motion, the particle charge executes a helical motion around the path of centre of mass and therefore the path of the particle appears as blurred. In complex vector space, the position and momentum of the particle can be considered as complex vectors. In the complex vector formalism, the energy of the classical harmonic oscillator was shown to be equal to that of a quantum particle oscillator and a relation between particle mass and spin angular momentum was derived. ${ }^{23}$ Further, it has been proved that the relativistic effects that we observe are due to the internal complex rotation at particle level.

The aim of this review article is to give a short account of the extended particle structure in complex vector space and to study the origin of relativistic effects due to a charged particle motion in the presence of zeropoint field. The mathematical apparatus for understanding the complex vector approach to the oscillating particle in zeropoint field is introduced in section 2. The extended particle structure in complex vector space is discussed in section 3 . The special relativity of extended particle is discussed in section 4 and discussion of the theoretical formulation is given in section 5 .

\section{Algebra of complex vectors}

It is well known that the quantities like angular velocity, torque remain same under space inversion. Such quantities are handled by introducing an ad-hoc axial vector which is decreed to be invariant. In vector algebra, the cross product exists only in three dimensions, in two dimensions it cannot be defined and in four dimensions the concept of a vector orthogonal to the plane defined by a pair of vectors is not unique. Such limitations are simply removed by introducing a bivector product in geometric algebra or Clifford algebra and it is regarded as a superior algebra over vector algebra. ${ }^{28,29} \mathrm{~A}$ detailed account of geometric algebra and its applications is given by Doran and Lasenby ${ }^{30}$ and it can be shown that the complex vector algebra is a sub-algebra of geometric algebra. The physical space we visualize is the three dimensional Euclidean space. Let $\boldsymbol{a}$ and $\boldsymbol{b}$ are two arbitrary vectors. The geometric product of these two vectors is expressed as a sum of scalar and bivector products in the following form.

$$
a b=a . b+a \wedge b
$$

The scalar or symmetric product is defined as

$$
\boldsymbol{a} \cdot \boldsymbol{b}=\frac{1}{2}(\boldsymbol{a} \boldsymbol{b}+\boldsymbol{b a})
$$

The bivector or asymmetric product is defined as

$$
\boldsymbol{a} \wedge \boldsymbol{b}=\frac{1}{2}(\boldsymbol{a b}-\boldsymbol{b a})
$$

A bivector defined above represents an oriented plane and it is invariant under space inversion. In geometric algebra, changing the order of vectors is called reversion operation which is indicated by an over bar. For example,

$$
\bar{a} \bar{b}=b a=a \cdot b-a \wedge b
$$

A wedge product of three vectors $\boldsymbol{a}, \boldsymbol{b}$ and $\boldsymbol{c}$ is called a trivector, $\boldsymbol{a} \wedge \boldsymbol{b} \wedge \boldsymbol{c}$. The three dimensional space can be spanned by considering a set of unit right handed orthogonal basis vectors $\left\{\sigma_{k} ; k=1,2,3\right\}$. The geometric product of vectors $\sigma_{1}, \sigma_{2}$ and $\sigma_{3}$ is called a pseudoscalar, $i=\sigma_{1} \sigma_{2} \sigma_{3}$ and it represents a unit oriented volume. The geoemetric 
products $\sigma_{i} \sigma_{j}(i \neq j)$ form unit bivectors which represent oriented orthogonal planes of unit magnitude and these are also obtained by multiplying $\sigma_{k}$ with pseudoscalar, $\mathrm{B}_{k}=\sigma_{i} \sigma_{j}=i \sigma_{k}$. The set of elements $\left\{1, \sigma_{k}, \mathrm{~B}_{k}, i ; k=1,2,3\right\}$ form geometric algebra of Euclidean space. A general element in geometric algebra is called a multivector which is a sum of a scalar, vector, bivector and trivector.

$$
M=\alpha+\boldsymbol{a}+\boldsymbol{i b}+\boldsymbol{i} \delta,
$$

where $\alpha$ and $\delta$ are scalars, $\boldsymbol{a}$ and $\boldsymbol{b}$ are vectors and $\boldsymbol{i} \delta$ is a trivector.

We define a complex vector as a sum of a vector and a bivector.

$$
Z=\boldsymbol{a}+\boldsymbol{i b}
$$

Here, the bivector $\boldsymbol{i} \boldsymbol{b}$ represents an oriented plane with counterclockwise rotation and the vector $\boldsymbol{a}$ lies in the plane of $\boldsymbol{i} \boldsymbol{b}$. The definition of complex vector in (6) provides a geometric understanding of the orientation of plane of rotation in space. The complex conjugate of complex vector $Z$ is obtained by taking a reversion operation.

$$
\bar{Z}=\boldsymbol{a}-\boldsymbol{i b}(7)
$$

Notice the change of sign before bivector in (7) and the rotation in the plane $\boldsymbol{i} \boldsymbol{b}$ is clockwise. The square of complex vector $Z$ is a complex scalar.

$$
Z^{2}=(\boldsymbol{a}+\boldsymbol{i} \boldsymbol{b})(\boldsymbol{a}+\boldsymbol{i} \boldsymbol{b})=a^{2}-b^{2}+2 \boldsymbol{i}(\boldsymbol{a} \cdot \boldsymbol{b})
$$

The square of the complex conjugate $\bar{Z}$ is also a complex scalar.

$$
\bar{Z}^{2}=(\boldsymbol{a}-\boldsymbol{i} \boldsymbol{b})(\boldsymbol{a}-\boldsymbol{i} \boldsymbol{b})=a^{2}-b^{2}-2 \boldsymbol{i}(\boldsymbol{a} \cdot \boldsymbol{b})
$$

The product of a complex vector with its conjugate contains scalar and vector parts. The products $Z \bar{Z}$ and $\bar{Z} Z$ are written as

$$
\begin{aligned}
& \bar{Z} Z=a^{2}+b^{2}+2 \boldsymbol{i}(\boldsymbol{a} \wedge \boldsymbol{b}) \\
& Z \bar{Z}=a^{2}+b^{2}-2 \boldsymbol{i}(\boldsymbol{a} \wedge \boldsymbol{b})
\end{aligned}
$$

Since, $\boldsymbol{i}$ is a pseudoscalar which commutes with all vectors in three dimensional space, the quantity $2 \boldsymbol{i}(\boldsymbol{a} \wedge \boldsymbol{b})$ is a vector and it is normal to the orientation of the bivector $\boldsymbol{a} \wedge \boldsymbol{b}$. The scalar part in (10) represents the scalar product of $\bar{Z}$ and $Z, \bar{Z} \cdot Z=a^{2}+b^{2}$ and the vector part represents the bivector product of $\bar{Z}$ and $Z, \bar{Z} \wedge Z=2 \boldsymbol{i}(\boldsymbol{a} \wedge \boldsymbol{b})$. A detailed discussion on complex vector algebra and complex vector space is discussed in the reference. ${ }^{24}$

\section{Extended particle structure in complex vector space}

As discussed in the introduction the internal oscillations of the electron due to fluctuating zeropoint field led to the consideration of particle extended structure. In this section, first we study these internal oscillations or zitterbewegung motion of Dirac electron as explored by Schrödinger ${ }^{2,31}$ and later we define the complex position coordinate describing both centre of mass and centre of charge of the extended electron. The Dirac Hamiltonian for the free electron can be expressed in the following form.

$$
H=c \alpha \cdot p+m c^{2} \alpha_{0},
$$

Where the set of matrices $\left\{\alpha_{\mu} ; \mu=0,1,2,3\right\}$ are known as Dirac matrices and satisfy the following anti-commutation relations.

$$
\left\{\alpha_{i}, \alpha_{j}\right\}=\alpha_{i} \alpha_{j}+\alpha_{j} \alpha_{i}=2 \delta_{i j} 1 ;\left\{\alpha_{i}, \alpha_{0}\right\}=0 \text { and } \alpha_{0}^{2}=1
$$

In quantum mechanics, the well known commutation relation for position $x$ and momentum $p$ operators of the electron is given by $[x, p]=i \hbar$. In the Heisenberg picture, the time derivative of an operator $A$ is given by

$$
\frac{d A}{d t}=\frac{i}{\hbar}[H, A] .
$$

Substituting the position operator $x$ for $A$ in the above equation yields the relation $\dot{x}=c \alpha$. Similarly, replacing $A$ in (14) by operator $\alpha$ gives

$$
-i \hbar \dot{\alpha}=2 H\left(\alpha-c H^{-1} p\right)=2 H \eta,
$$

where $\eta=\alpha-c H^{-1} p$. Since the time derivative $\dot{\eta}=\dot{\alpha},(15)$ can be written in the form

$$
\dot{\eta}=\frac{i 2 H}{\hbar} \eta
$$

The solution of this first order differential equation is easily found to be

$$
\eta(t)=\eta(0) \exp \left(\frac{i 2 H t}{\hbar}\right)
$$
have

where $\eta(0)=\alpha(0)-c H^{-1} p$. Now, using the relation $\dot{x}=c \alpha$ we

$$
\dot{x}=c^{2} H^{-1} p+\left[c \alpha(0)-c^{2} H^{-1} p\right] \exp \left(\frac{i 2 H t}{\hbar}\right) .
$$
form

Further integration gives the required position coordinate in the

$$
x(t)=(x(0)+\dot{x} t)+\frac{1}{2} i \hbar\left[c \alpha(0)-c^{2} H^{-1} p\right] H^{-1} \exp \left(\frac{i 2 H t}{\hbar}\right) .
$$

This equation contains two parts: the first part represents the position of centre of the particle and the second the oscillations of the particle charge. These internal oscillations are known by the name zitterbewegung. The cause of such oscillations is attributed to the electromagnetic fluctuating random zeropoint field and the frequency of these internal oscillations $\omega=2 m c^{2} \hbar^{-1}$. The average of all such internal oscillations leads to blurred paths of electrons and gives rise to uncertainty in position. We identify the first part on left of (19) as the centre of mass position vector, $\boldsymbol{x}(t)=\boldsymbol{x}(0)+\dot{\boldsymbol{x}} t$ and replacing the unit imaginary in the second term by pseudoscalar we represent the position of centre of charge $\xi(t)=\xi(0) \exp \left(i \omega_{0} t\right)$, where we have chosen $\xi(0)=\frac{1}{2} \hbar\left[c \alpha(0)-c^{2} H^{-1} p\right] H^{-1}$, and $\omega_{0}=\frac{2 H}{\hbar}$. Now, one can construct a position complex vector from (19) in the form

$$
X=x(t)+i \xi(t) .
$$

The complex vector defined above is similar to the coordinate introduced by Barut and Zanghi. ${ }^{34}$ However, they did not consider the bivector $i \xi$. The complex vector $X$ gives an additional picture of the structure of a charged particle with internal oscillations represented 
by rotations in the complex plane and the radius of circular motion of centre charge around centre of mass is denoted by $\xi(0)$. Thus the complex vector $X$ describes the extended structure of an electron or a charged elementary particle under consideration. When the frame of reference is positioned at the centre of mass point, i.e., the particle is at rest, only the circular motion of charge is observed. When the particle is observed from an arbitrary frame, the centre of mass moves with certain velocity and the charge appears to take helical motion and the proper time is attached with the centre of mass point. The angular momentum of internal circular motion is called spin angular momentum of the charged particle. ${ }^{23}$ Therefore, the point particle concept or rigid body with charge distribution of an elementary charged particle is denied and we accept the extended structure of charged particle containing the internal oscillation or rotation which is a consequence of zeropoint field present throughout universe.

\section{The special relativity of an extended particle}

In this section we shall show that the helical motion of charge centre of an elementary particle is the root cause of relativistic effects observed from an arbitrary frame of reference. The calculation of time dilation formula from helical motion of charge was first derived by Cavelleri. ${ }^{32}$ Here, we use the complex vector approach to show the origin of special relativity as a consequence of extended particle structure. ${ }^{23}$ The velocity complex vector of an extended charged particle is obtained by differentiating (20) with respect to time.

$$
\boldsymbol{U}=\frac{d \boldsymbol{X}}{d t}=\boldsymbol{v}+\boldsymbol{i} \boldsymbol{u}
$$

where, $\boldsymbol{v}=d \boldsymbol{x} / d t$ and $u=d \xi / d t$ are the velocity of centre of mass and the internal instantaneous velocity respectively. The magnitude of internal velocity is equal to the velocity of light which is the velocity of electron in Dirac theory. In the complex vector $X$, the vector $\xi$ is considered orthogonal to $\boldsymbol{x}$ and hence in (21) the velocity $\boldsymbol{u}$ is orthogonal to the particle translational velocity $\boldsymbol{v}$. A reversion operation on velocity complex vector $\boldsymbol{U}$ gives its conjugate,

$$
\bar{U}=\boldsymbol{v}-\boldsymbol{i u} .
$$

Now, we take the scalar product of complex vectors $\boldsymbol{U}$ and $\overline{\boldsymbol{U}}$.

$$
\overline{\boldsymbol{U}} \cdot \boldsymbol{U}=v^{2}+u^{2} .
$$

The above equation shows the superposition of internal velocity on the translational velocity. In the particle rest frame the translational velocity $\boldsymbol{v}=0$ and the internal velocity is equal to velocity of light, $u=c$ and then the magnitude $|U|=u=c$. However, when the particle is observed from an arbitrary frame different from the rest frame of the particle, the particle motion contains both translational and internal rotational motion and the product $\overline{\boldsymbol{U}} \cdot \boldsymbol{U}=c^{2}$. Now, the particle internal velocity can be expressed in the form

$$
u=\left(c^{2}-v^{2}\right)^{1 / 2}=c\left(1-\beta^{2}\right)^{1 / 2}=c \gamma^{-1},
$$

where $\beta=-\frac{\boldsymbol{v}}{\delta}$ and the factor $\gamma$ is the time dilation factor. If we attach a rest frame to the centre of mass position, the angular frequency of charge rotation is equal to the ratio between the velocity $c$ and radius of rotation.

$$
\omega_{0}=\frac{c}{\xi}
$$

However, when observed from an arbitrary frame, the angular frequency $\omega$ will be equal to the ratio between $u$ and $\xi$.

$$
\omega=\frac{u}{\xi}=\omega_{0} \gamma^{-1}
$$

In general $u$ and $\xi$ are vectors and the angular frequency is a bivector. Thus we have $-i \omega=\frac{u}{\xi}$ or $-i \omega \wedge \xi=u$ and the equation (26) is
expressed in the form

$$
\Omega=\Omega_{0} \gamma^{-1},
$$

where $\Omega=-i \omega$ and $\Omega_{s}=-i \omega_{0}$. When the particle motion is observed from an arbitrary frame of reference, equation (27) elucidates that the angular frequency of rotation decreases by a factor $\gamma^{-1}$. In other words, if the charge centre moves over a distance along the rotation path in $d t$ time, the proper time interval along the path of centre of mass will be $d \tau=\gamma^{-1} d t$ which represents the time dilation formula. It can be proved in a similar fashion when the particle centre of mass point moves over a proper distance $d x^{\prime}$, the distance observed in the arbitrary frame would be the length contraction formula $d x^{\prime}=\gamma d x$.

From the above logical relativistic formulation, we represent the relative velocity of a charged particle by $\gamma \boldsymbol{v}$ and the relativistic momentum $\boldsymbol{p}=\gamma \boldsymbol{m} \boldsymbol{v}$. In the rest frame of the particle, it may be noted that this momentum is zero. Now, we construct the momentum complex vector in the following form.

$$
P=p+i \pi,
$$

where $\pi=m \boldsymbol{u}$ is the internal momentum. Taking a reversion operation on this complex vector gives

$$
\bar{P}=p-i \pi \text {. }
$$

Now, taking the scalar product of $P$ and $\bar{P}$ and multiplying it by $c^{2}$ gives the relativistic energy relation in terms of momentum.

$$
E^{2}=P \cdot \bar{P} c^{2}=p^{2} c^{2}+m^{2} c^{4}
$$

The above complex vector approach univocally suggests that the special theory of relativity emerges from the internal complex rotations in the local space. Since the very presence of zeropoint field is the cause of internal complex rotation of the particle, the relativistic effects are then attributed to the zeropoint fields. In general, this method of finding relativistic effects is applicable to all charged massive spin half particles or fermions.

The particle spin is the zeropoint angular momentum associated with the zeropoint energy. The internal zeropoint angular momentum in the rest frame of the particle represents the spin bivector ${ }^{23} S=\xi \wedge \pi$. For a particle moving in a curved path, the orbital angular momentum is defined by a bivector $x \wedge p$. Expanding the bivector product $X \wedge \bar{P}$ gives $^{25}$

$$
X \wedge \bar{P}=x \wedge p+\xi \wedge \pi+i(\xi \wedge p)-i(x \wedge \pi) .
$$

The first term on right of (31) is the bivector angular momentum, the second term represents internal angular momentum or the spin and the other two terms are vectors. The bivector $\xi \wedge p$ represents an oriented plane perpendicular to spin plane. Therefore, combining the second and third terms on right of (31) we obtain a complex vector in the form 


$$
S_{t}=S+\mathbf{i} S_{L}
$$

where, $S_{L}=\xi \wedge p$. Using the magnitude of radius of rotation $|\xi|=\hbar(2 m c)^{-1}$ and choosing the vector $\xi$ along direction of unit vector $\sigma_{2}$, the bivector $S_{L}$ can be expressed as

$$
S_{L}=i \sigma_{2} \gamma \beta \frac{\hbar}{2}
$$

Now, considering the scalar product $S_{t} \cdot \bar{S}_{t}=-\mathrm{S}_{0}^{2}$, we find the magnitude of spin

$$
\mathrm{S}_{0}^{2}=-S_{t} \cdot \bar{S}_{t}=-\left(S^{2}+S_{L}^{2}\right)=\gamma^{2} S^{2}
$$

$$
\text { Or }\left|S_{0}\right|=\gamma|S|
$$

The relativistic expression for the particle spin is then obtained by multiplying (34) on both sides by $i / \sigma_{3}$.

$$
S_{0}=i \sigma_{3}\left|S_{0}\right|=\gamma S
$$

Thus, when the particle centre of mass moves with a velocity $\boldsymbol{v}$, the magnitude of spin appears to be increased by a Lorentz factor.

From the above considerations, a charged particle at rest is not actually at rest but the centre of charge oscillates with certain frequency. The frequency of these oscillations will be dictated by the fluctuations of the zeropoint field. The stochastic average of these internal oscillations of the particle describes circular motion in complex vector space and the particle oscillations may be considered as simple harmonic and such simple harmonic oscillator absorbs energy from the zeropoint field. The local properties of the charged particle limit the spectral response of the zeropoint field and the particle absorbs energy at single cut-off frequency. In the purview of stochastic electrodynamics with spin, in the rest frame of the particle, it has been shown that the energy associated with the particle oscillator is equal to zitterbewegung energy. ${ }^{25}$

Initially, one can assume that a charged particle may not have any mass and the interaction of such mass less particle with zeropoint field causes local oscillations of the particle charge. The stochastic average of all such rotations can be expressed as complex rotation of charge. The zeropoint energy associated with such complex rotation was shown to be equal to particle mass. ${ }^{25}$ In the rest frame of the particle, a relation between particle spin and mass was derived in the reference. ${ }^{23}$

$$
m c^{2}=\Omega_{0} . \mathrm{S},
$$

When the particle is observed from an arbitrary frame, the particle centre of mass appears to move with certain velocity and according to (27) and (35) the angular frequency bivector decreases by a factor $\gamma^{-1}$ and the spin changes by a factor $\gamma$. Therefore, the product $\Omega$.S does not change with the particle motion and therefore, the particle mass is a constant of motion. However, we do not consider here the mass correction due to the particle centre of mass motion in the zeropoint fields and it will be explored elsewhere.

\section{Discussion}

Acharged massive particle like electron is considered as an oscillator in the presence of fluctuating electromagnetic zeropoint field. It is well known that the Dirac electron executes zitterbewegung motion and it is attributed to the oscillations of the particle in zeropoint field. The presence of zeropoint field was first perceived by Nernst in 1916 and proposed that the difference between field and matter oscillators is inadmissible because, in thermal contact, these systems attain equilibrium. ${ }^{33}$ The zeropoint radiation pervades the entire universe and found to be electromagnetic, homogeneous, isotropic, and Lorentz invariant and therefore it is known as classical in its nature. Though the direct observation of zeropoint field is not possible, its effects on various quantum and classical systems have been theoretically found and experimentally observed. According to Puthoff $\mathrm{f}^{34}$ the zeropoint field is dynamically generated by the motion of charged particles throughout the universe and at the same time the particles are under the influence of zeropoint fluctuations and the process is regenerative and leads to the generation of grand ground state of the universe. A charged massive particle like electron is considered as an oscillator in the presence of fluctuating electromagnetic zeropoint field. A stochastic average of all such oscillations of electron in different directions in space can be considered as a complex rotation in complex vector space. The charged particle is then assumed to possess extended structure with its centre of mass and centre of charge separated by an average distance equal to half the Compton wave length. The position of such extended particle is expressed as a complex vector. When the particle centre of mass is in motion, we find the relativistic effects are due to the particle extended structure. Since the particle structure is due to the presence of zeropoint field, the observed relativistic effects of particles are attributed to the ubiquitous presence of zeropoint field. Further, we have explained that the particle mass arises from the interaction of particle oscillator with zeropoint field.

\section{Acknowledgments}

None.

\section{Conflict of interest}

Author declares that there is no conflict of interest.

\section{References}

1. Sakurai JJ. Advanced quantum Mechanics. Pearson Education. 2007;128.

2. Barut AO, Bracken AJ. Zitterbewegung and the internal geometry of electron. Phys Rev D. 1981;23:2454.

3. Huang K. On the zitterbewegung of Dirac electron. Am $J$ Phys. 1952;20(8):479-484.

4. Mathisson M. Neuemekhanikmaterietter system. Acta Phys Pol. 1937;6:163-200.

5. Weyssenhoff J, Raabbe A. Relativistic dynamics of spin fluids and spin particles. Acta Phys Pol. 1947;9:7-18.

6. Horvathy PA. Mathisson's spinning electron: Non commutative mechanics and exotic Galilean symmetry 66 years ago. Acta Phys Pol. 2003;34:2611-2622.

7. Barut AO, Zanghi AJ. Classical model of the Dirac electron. Phys Rev Lett. 1984;52:2009-2012. 
8. Hestenes D. Spin and uncertainty in the interpretation of quantum mechanics. Am J Phys. 1979;47: 399-415.

9. Hestenes D. Zitterbewegung in quantum mechanics. Found Phys. 2010;40:1-54.

10. Rivas M. Kinematical theory of spinning particles. Kluwer Academic publishers. Dordrecht. 2001.

11. Gabrielse G, Hanneke D, Kinoshita T, et al. New Determination of the Fine Structure Constant from the Electron $g$ Value and QED. Phys Rev Lett. 2006;97:030802.

12. Gouanère M, Spighel M, Gaillard MJ Roussel, Experimental observation compatible with the particle internal clock. Annales de la foundation Louis de Broglie. 2005;30:109-114.

13. Hofer WA. An extended model of electrons: experimental evidence from high-resolution scanning tunneling microscopy. J Phys Conference Series. 2012;361: 012023.

14. Boyer TH. Random electrodynamics: The theory of classical electrodynamics with classical electromagnetic zeropoint radiation. Phys Rev D. 1975; 11: 790-808.

15. Boyer TH. A brief survey of stochastic electrodynamics. In: Foundations of Radiation Theory and Quantum Electrodynamics. Springer. 1980;49-63.

16. Daniel C Cole. Reviewing and extending some recent work on stochastic electrodynamics. In: Essays on the formal aspects of electromagnetic theory. A Lakhtakia, editor. World Scientific publ Co. 1993;501-532.

17. Lde la Pena, Cetto AM. Quantum Dice: An Introduction to Stochastic Electrodynamics. Dordrecht. 1996.

18. Rueda A, Cavalleri G. Zitterbewegung in stochastic electodynamics and implications on a zero-Point field acceleration mechanism. Nuovo Cimento. 1983;6:239-260.

19. Rueda A. Stochastic electrodynamics with particle structure part I: Zeropoint induced Brownian behaviour. Found Phys Lett. 1993;6:75-101.

20. Rueda A. Stochastic electrodynamics with particle structure part II: Towards zero-point induced wave behaviour. Found Phys Lett. 1993;6:139-166.
21. Cavalleri G, Barbero F, Bertazzi G, et al. A quantitative assessment of stochastic electrodynamics with spin (SEDS): Physical principles and novel applications. Front Phys China. 2010;5:107-122.

22. Bosi L, Cavalleri G, Barbero F. Review of stochastic electrodynamics with and without spin. In: Proceedings of Physical interpretation of relativity theory (PIRT XI), UK: London. 2008;12-15.

23. Muralidhar K. Complex vector formalism of harmonic oscillator in geometric algebra: Particle mass, spin and dynamics in complex vector space. Found Phys. 2014;44:266-295.

24. Muralidhar K. Algebra of complex vectors and applications in electromagnetic theory and quantum mechanics. Mathmatics. 2015;3:781-842.

25. Muralidhar K. Mass of a charged particle with complex structure in zerpoint field. Progress in physics. 2016;12:224-230.

26. Muralidhar K. Classical origin of quantum spin. Apeiron. 2011;18(2):146160.

27. Muralidhar K. The spin bivector and zeropoint energy in geometric algebra. Adv Stud Theor Phys. 2012;6:675-686.

28. Hestenes D. Oersted Medal Lecture 2002: Reforming the Mathematical Language of Physics. Am J Phys. 2003;71:104-121.

29. Hestenes D. Space-time algebra, Gordon and Breach Science Publishers. New York. 1966.

30. Doran C, Lasenby A. Geometric algebra for physicists, Cambridge university press, Cambridge. 2003.

31. Sidharth B. Revisiting Zitterbewegung. Int $J$ Theor Phys. 2009;48(2):497-506.

32. Cavalleri G. $\hbar$ derived from cosmology and origin of special relativity and QM. Nuovo Cimento. 1997;112:1193-1205.

33. Boyer TH. The classical vacuum. Sci Am. 1985;253:70-78.

34. Puthoff HE. The source of vacuum electromagnetic zeropoint energy. Phys Rev A. 1989;40:4857. 